\title{
Closed reduction and immobilization of displaced distal radial fractures. Method of choice for the treatment of children?
}

\author{
D. S. Wendling-Keim $\cdot$ B. Wieser $\cdot$ H.-G. Dietz
}

Received: 20 June 2014 / Accepted: 24 November 2014 / Published online: 19 December 2014

(C) Springer-Verlag Berlin Heidelberg 2014

\begin{abstract}
Purpose The therapy of distal radial fractures in children is expected to be as non-invasive as possible but also needs to deliver the definite care for gaining optimal reduction and stabilizing the fracture. Therefore, closed reduction and immobilization is competing with routine Kirschner wire fixation. The aim of our study was to investigate if closed reduction and immobilization without osteosynthesis can ensure stabilization of the fracture.

Methods We chose a retrospective study design and analyzed 393 displaced distal radial fractures in children from 1 to 18 years with open epiphyseal plates studying medical files and X-rays. The Pearson's $\chi^{2}$ test was applied. Statistical analysis was performed using IBM SPSS Statistics 20.0. Statistical significance was set at an alpha level of $P=0.05$.

Results Of these studied fractures 263 cases were treated with closed reduction and immobilization. Only 38 of these needed secondary interventions, 28 of these underwent reduction after redisplacement and ten patients received secondary Kirschner wire fixation. The last follow-up examination after 4-6 weeks revealed that $96.4 \%$ of fractures initially treated with closed reduction and immobilization were measured within the limits of remodeling. 104 of the studied fractures were treated with cast immobilization alone when displacement was expected to correct due to remodeling. Here $22.1 \%$ of patients needed secondary reduction. Furthermore, primary Kirschner wire fixation was performed in only 25
\end{abstract}

D. S. Wendling-Keim $(\varangle) \cdot$ B. Wieser $\cdot$ H.-G. Dietz Pediatric Surgery, Dr. von Haunersches Kinderspital, Ludwig-Maximilian-University of Munich, Lindwurmstr. 4, 80337 Munich, Germany

e-mail: daniellewendling@gmx.net children with unstable fractures and only one received further treatment. Interestingly, operative reports of primary closed reduction revealed that repeated maneuvers of reduction as well as residual displacement are risk factors for redisplacement.

Conclusion For the treatment of displaced distal radial fractures in children closed reduction and immobilization can be considered the method of choice. However, for cases with repeated reduction maneuvers or residual displacement we recommend primary Kirschner wire fixation to avoid redisplacement.

Level of evidence Retrospective comparative study, Level III

Keywords Distal radial fracture - Closed reduction . Kirschner wire fixation $\cdot$ Epiphyseal plate

\section{Introduction}

Distal radial fractures are among the most common injuries in children [1,2], and fractures at the metaphysis are especially prevalent $[3,4]$. Closed reduction and cast immobilization play a major role in the treatment of these fractures in children [4, 5]. However, various rates of unacceptable loss of reduction have been described in the literature [612] and therefore many studies have been undertaken to identify the factors which increase the risk of redisplacement. Among the main determinants that have been found are the residual displacement after initial reduction $[6,10$, 13] and the initial complete displacement of the radius [9, $10,14,15]$. Since the risk of redisplacement can be significantly reduced by the use of percutaneous pin fixation $[8$, 11] the initial use of Kirschner wires has been debated for fractures with an increased risk for loss of reduction [10, 
15-17]. However, no agreement regarding a gold standard has been found to date.

Therefore, the aim of our study was to investigate the rate of redisplacement after closed reduction and cast immobilization on a large-scale analyzing 393 cases of displaced distal forearm fractures and hereby to determine if this regimen still can be accepted as the method of choice for this type of fracture in children. Furthermore, our goal was to identify factors that increase the risk of the complication of redisplacement and therefore determine the indication of initial Kirschner wire fixation instead of only cast immobilization after closed reduction.

\section{Materials and methods}

The Study was carried out retrospectively. Patients with displaced radial fractures aged $0-18$ years with epiphyseal plates who were treated in the pediatric surgery clinic at the Dr. von Haunersches Kinderspital from 2005 through 2011 were selected from the hospital's electronic radiologic archive. Patients with Salter/Harris 3 and Salter/ Harrys 4 fractures as well as stress fractures, pathological fractures, refractures, any known bone disease or pubertas praecox and tarda were excluded from the study. Fracture treatment and outcome were analyzed considering angulation and translation as well as involvement of the epiphysis, concomitant fractures and volar or dorsal displacement and therefore the use of fracture classification systems was not incorporated in this study.

Fractures were treated with cast immobilization alone or closed reduction and cast immobilization or Kirschner wire fixation or, although very rarely, with ESIN (elastic stable intramedullary nailing) according to the degree of displacement, the stability after reduction and the decision of the surgeon on call. Closed reduction was performed under general anesthesia with one exception due to cystic fibrosis. A C-arm was used to control the position of the fragments. Immobilization was achieved by the fitting of long-arm plaster- or fiberglass splints.

All radiographs and patient records including operation reports were analyzed. Optimal reduction was defined as angulation of less than $5^{\circ}$ and translation of less than corticalis width. Redisplacement of any degree, number of interventions and complications were registered as well as demographic data. Any increase in angulation or translation after the initial treatment was registered as redisplacement and further analyzed.

Limits of remodeling were considered $\leq 30^{\circ}$ angulation and $\leq$ shaft width translation for children under 12 years of age and $\leq 15^{\circ}$ angulation and $\leq 1 / 2$ shaft width translation for children aged 12 years or older, respectively. age distribution of patients with displaced distal radial fractures $n=393$

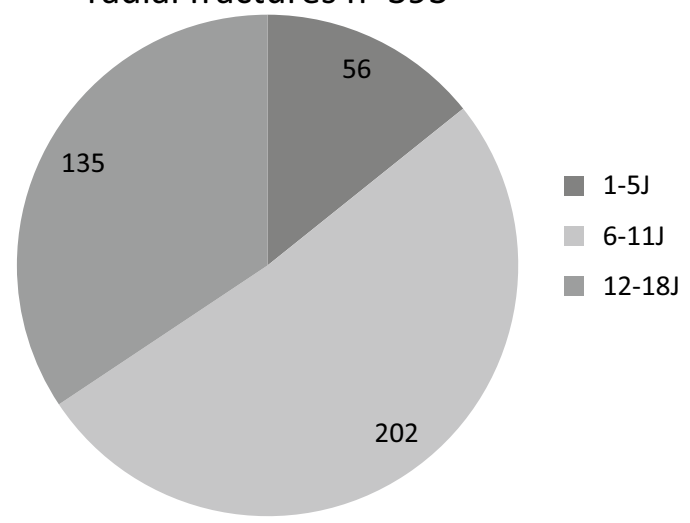

Fig. 1 The age distribution demonstrates the majority of patients in the study were between 6 and 11 years of age

Table 1 Frequency within the different age groups

\begin{tabular}{ll}
\hline Age group (years) & $\begin{array}{l}\text { Redisplacement } \\
\text { rate }(\%)\end{array}$ \\
\hline $1-5$ & 45.3 \\
$6-11$ & 40.5 \\
$12-18$ & 31.8 \\
\hline
\end{tabular}

The pearson's $\chi^{2}$ test was applied. Statistical analysis was performed using IBM SPSS Statistics 20.0. Statistical significance was set at an alpha level of $P=0.05$.

\section{Results}

383 patients with displaced distal radial fractures were detected during the study period from 2005 through 2011 yielding 393 cases since in ten patients two fractures of the distal radius occurred either at the same time in different arms or at different points of time in the same arm but at different locations within the distal radius.

The mean age was 9.8 years, $72.5 \%$ of patients were male and $27.5 \%$ female. The age distribution is shown in Fig. 1. Of all cases $82.1 \%$ extension fractures and $17.9 \%$ flexion fractures were noted.

Of all displaced distal radial fractures secondary displacement was found in 143 cases, respectively, $36.4 \%$. However, any displacement after the initial therapy was registered in this study to avoid any bias and only 56 of these absolutely redisplaced fractures needed further treatment. Redisplacement resulting from a new trauma or from a defect cast was excluded. Analysis of the age pattern showed 
Table 2 Redisplacement detected mainly within the first week after primary treatment

\begin{tabular}{lcl}
\hline Time of detection of redisplacement & Occurrence & Rate $(\%)$ \\
\hline 1 day & 2 & 1.4 \\
1 week & 82 & 57.3 \\
2 weeks & 13 & 9.1 \\
3 weeks & 14 & 9.8 \\
4 weeks & 21 & 14.7 \\
5 weeks & 3 & 2.1 \\
6 weeks & 7 & 4.9 \\
Unknown due to treatment in external & 1 & 0.7 \\
$\quad$ hospital & & \\
Total & 143 & 100 \\
\hline
\end{tabular}

that redisplacement was more frequent in younger patients pointing to the fact that the choice of treatment seems to be strongly influenced by the growth-dependent correction potential (Table 1). Furthermore, redisplacement was mainly detected one week after trauma or reduction (Table 2).

Furthermore, the differentiation of fracture types revealed a tendency of increased redisplacement rate in radial fractures with concomitant ulnar fractures $(P=0.051)$ as compared to isolated radial fractures whereas involvement of the ulnar styloid processus did not have any effect. Also, Salter/Harris 1 and 2 fractures displayed a significantly lower redisplacement frequency compared to fractures that were more located further proximal which is related to the increased instability in the more proximal fractures $(P=0.005)$. Additionally, fractures with dorsal displacement redisplaced more often than volar fractures $(P=0.003)$, Of the analyzed 393 displaced distal radial fractures 263 cases were treated by closed reduction and immobilization while 104 fractures were only immobilized according to the expected growth-related correction potential depending on the age of the patient. Only 25 fractures, respectively, $6.3 \%$ were primarily fixated with Kirschner wires and due to individual decision ESIN was performed in one exceptional case (Fig. 2).

The majority of displaced distal radial fractures counting 263 cases, respectively, $66.9 \%$ were treated by closed reduction and immobilization. Above-elbow casts were applied. Angulation in these fractures ranged from $7^{\circ}$ to $54^{\circ}$ and translation from corticalis thickness to more than shaft width. Most patients were between 6 and 11 years of age (Fig. 3).

Of these patients who were treated with closed reduction 223 fractures, respectively, $84.8 \%$ did not need any further intervention, since $78.0 \%$ of redisplacements were measured to be less than $10^{\circ}$ and $96.4 \%$ of fractures displayed only slight residual displacements ranging within the limits of remodeling after closed reduction.

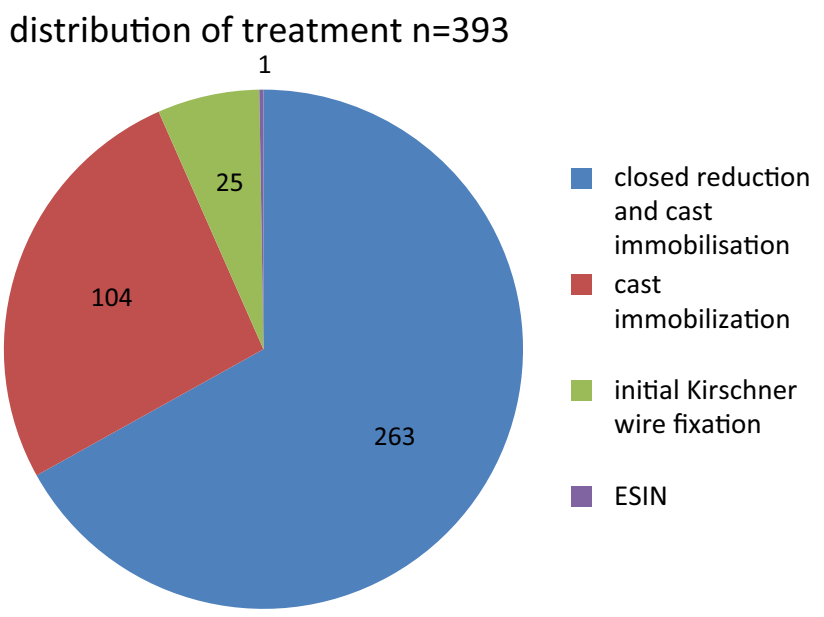

Fig. 2 It shows that most patients studied were treated with closed reduction and cast immobilization

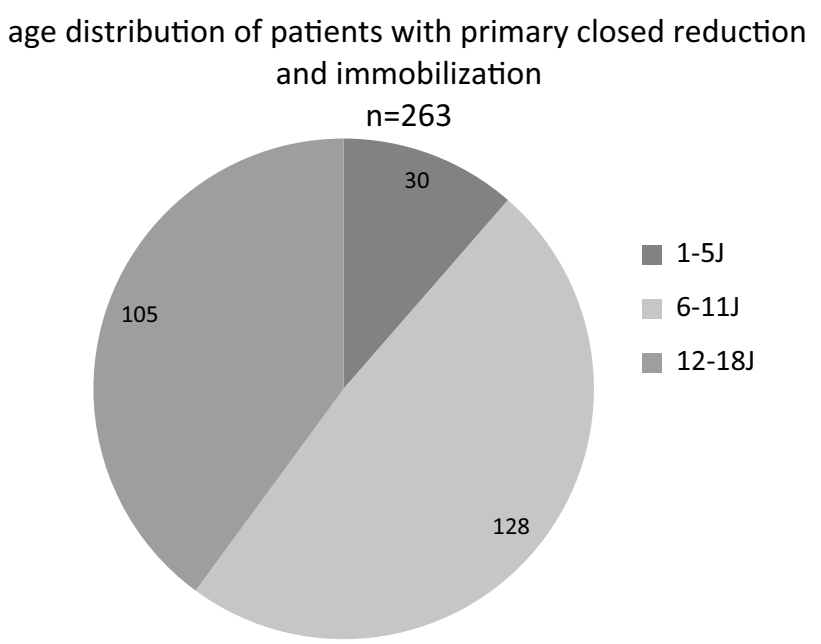

Fig. 3 Primary closed reduction and immobilization was applied for all age groups, however, the majority of patients was aged 6-11 years

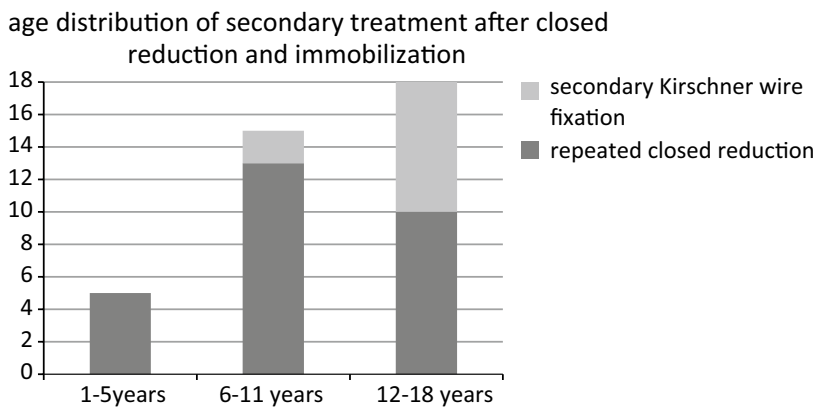

Fig. 4 The age distribution of patients needing secondary intervention after primary reduction demonstrates the dependence of frequency and type of secondary treatment on the remodeling potential according to the patient's age 


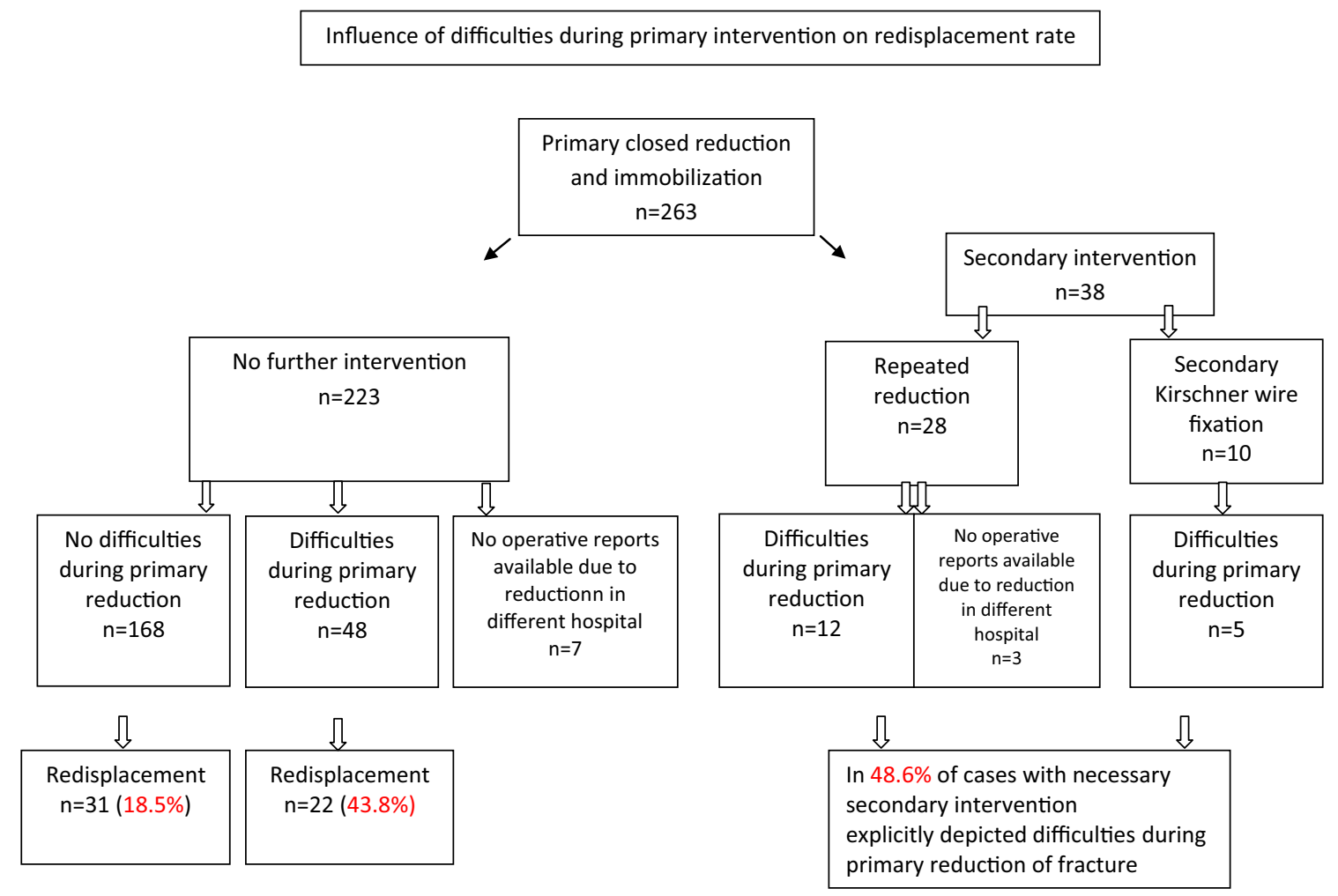

Fig. 5 Factors influencing redisplacement are shown here for patients primarily treated with closed reduction and immobilization

However, 38 fractures needed further treatment after closed reduction. 28 patients were treated with repeated closed reduction. Angulation and translation before repeated reduction were measured between $11^{\circ}$ and $44^{\circ}$ and $1 / 4$ and $1 / 2$ shaft width, respectively.

Furthermore, after primary closed reduction ten redisplaced fractures were secondarily fixated with Kirschner wires. Redisplacement angulation of $27^{\circ}-30^{\circ}$ and translation of $1 / 3$ shaft width or more were noted in these cases. Two more patients needed secondary Kirschner wires as well, however, these two cases were excluded from the analysis since their cast had been accidentally broken. Interestingly, $80 \%$ of patients with secondary Kirschner wire fixations were between 12 and 18 years of age and the remainining $20 \%$ were 6-11 years while no patient under 6 years received secondary Kirschner wires. Accordingly, patients aged 6-11 years with redisplacement after closed reduction was mainly treated with repeated reduction (Fig. 4).

To investigate the cause of spontaneous redisplacement after primary reduction in these 38 fractures we analyzed the operative reports. Operation reports of 35 cases were available since three fractures were not initially treated at our hospital. Interestingly, in $48.6 \%$ of these cases, we detected explicitly described problems during primary reduction consisting of necessary repetition of reduction and residual angulation of more than $5^{\circ}$ or translation of more than corticalis width after reduction. Furthermore, investigation of 216 of the 223 fractures without secondary intervention was performed, since seven were not treated initially at our hospital. The reports revealed that fractures that redisplaced after reduction, although within the limits of remodeling, had raised the above-mentioned problems significantly more often $(P=0.00029)$ than fractures that did not redisplace. While $18.5 \%$ of fractures without difficulties during reduction showed secondary displacement, $43.8 \%$ of cases with problems during primary reduction displaced again during follow-up (Fig. 5). We therefore, consider repeated reduction and residual angulation and translation $\left(5^{\circ}\right.$ or more and corticalis width or more, respectively) risk factors for redisplacement and therefore indication of primary Kirschner wire fixation. Regarding the outcome, $96.4 \%$ of 223 cases that were treated by primary closed reduction and immobilization alone were found to range within the limits of remodeling and $78.0 \%$ displayed an angulation of less than $10^{\circ}$. Furthermore, $89.3 \%$ of cases that needed secondary reduction and $100 \%$ of fractures with secondary Kirschner wire fixation after primary closed reduction were measured within the limits of remodeling while 64.3 and $91.7 \%$, respectively, showed a residual angulation of $10^{\circ}$ or less. 
age distribution of patients with primary immobilization $n=104$

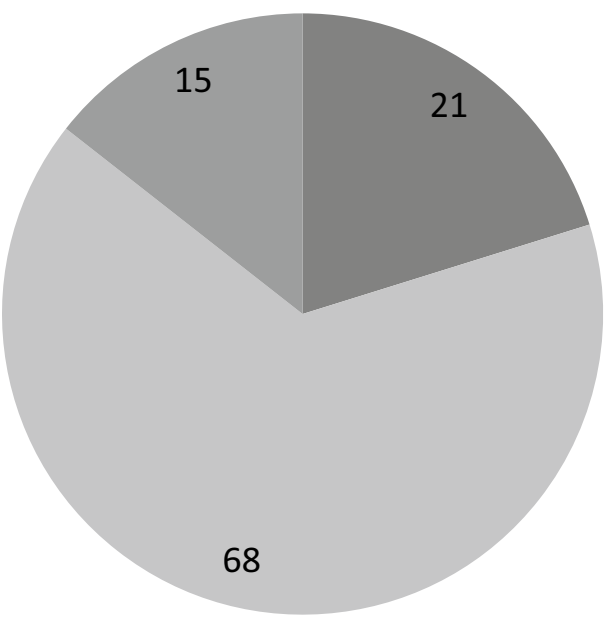

Fig. 6 The age pattern of patients primarily treated with immobilization is depicted here

Taken together, $14.4 \%$ of patients treated primarily with closed reduction and immobilization required further treatment, e.g., either secondary reduction in 28 cases or Kirschner wire fixation in ten cases. Eleven fractures displayed residual displacement beyond the limits of remodeling, however, limits were set very strictly. Maximal residual angulation was $40^{\circ}$ in the group of patients aged $1-5$ years, $35^{\circ}$ in the group of $6-11$ years and $26^{\circ}$ in patients from 12 to 18 years in the last follow up examination after 4-6 weeks.

Next, we investigated application of immobilization without closed reduction as initial treatment and found 104 fractures with a primary displacement degree ranging from $7^{\circ}$ to $48^{\circ}$. However, minor angulation was combined with severe translation. Children aged 8 years or younger received above-elbow casts while older patients were treated with underarm-casts. Only $14.4 \%$ of patients were older than 11 years in this treatment group (Fig. 6). Although in 51 fractures secondary displacement was detected (and additionally, ten fractures revealed an increase in displacement after another trauma or after accidentally breaking the cast and therefore, were excluded), only 18 of these needed secondary closed reduction and no fracture was fixated with Kirschner wires (Fig. 7). Three fractures were secondarily placed in Schede position. All other cases maintained their status or were expected to correct their displacement spontaneously due to remodeling. Taken together, 78 of 94 fractures $(83.0 \%)$ did not need any further treatment after cast immobilization and $86.2 \%$ were noted to be within limits of remodeling at the last follow-up examination after 4-6 weeks. age distribution of repeated reduction after primary immobilization

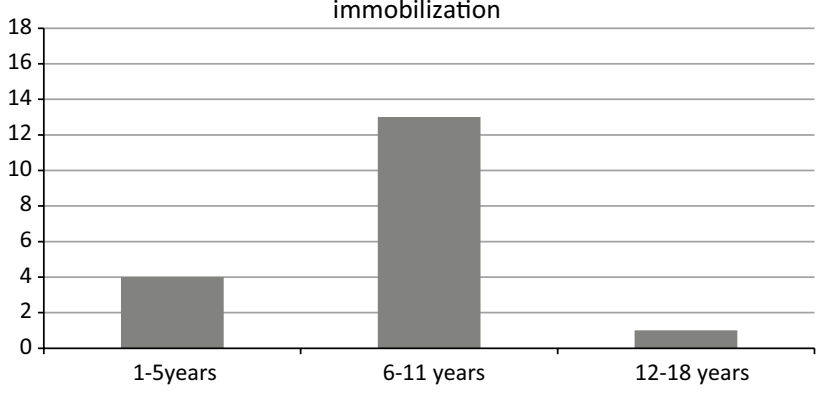

Fig. 7 Secondary intervention after primary cast immobilization alone consisted of secondary reduction only. Due to their remodeling potential, most patients were between 6 and 11 years of age

\section{age distribution of patients with Kirschner wire fixation $n=25$}

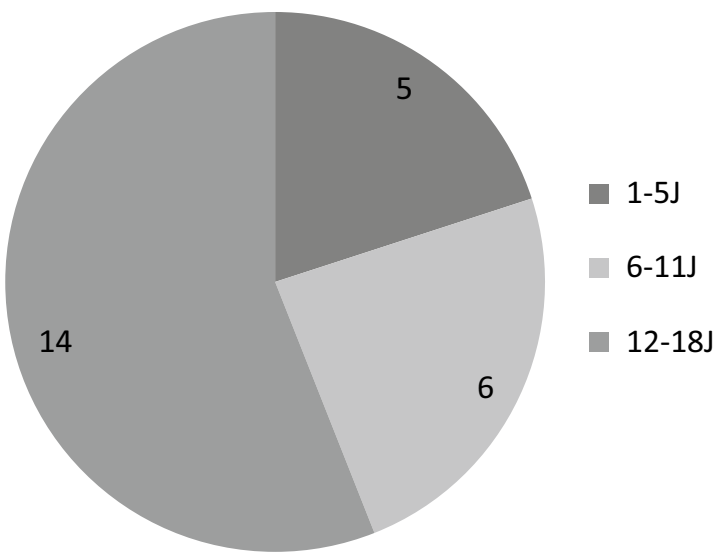

Fig. 8 More than half of patients treated with Kirschner wire fixation were older than 11 years of age

Summing up the cases of secondary reduction, 46 cases were registered, namely 18 times after primary immobilization alone and 28 times after primary closed reduction. Interestingly, of these secondary reductions only six fractures were unsuccessful due to consolidation within 1, 2 and 7 weeks. However, all other cases were effectively reduced secondarily.

Primary Kirschner wire fixation was performed in 25 patients corresponding to $6.11 \%$ of all studied cases. $80 \%$ of the patients treated with primary Kirschner wire fixation were older than 5 years (Fig. 8). Indication for this procedure was instability after closed reduction or necessity of open reduction intraoperatively. In the last follow-up examination, $92 \%$ of these fractures displayed a residual angulation of less than $10^{\circ}$ and no translation. Only one fracture redisplaced revealing an angulation of $15^{\circ}$. One patient, as an exception, however, displayed an increased angulation 
even in comparison to the initial X-ray and needed plating which is applied in children only very rarely and under special circumstances. Apart from this case complications were uncommon as one patient developed wound infection and one suffered from maceration of the skin in the area of the wire tip.

Of the studied fractures some had special features. Eight were open fractures, however, seven of them were rated Gustilo grade I and one Gustilo grade II. Furthermore 78 Salter-Harrys-II and 4 Salter-Harrys I fractures were counted as well as 14 green stick fractures and three plurifragmentary fractures. Interestingly, no significant difference regarding the redisplacement rate was detected in comparison to the fractures without special characteristics except for the fractures with involvement of the growth plate (Table 3).

Interestingly, involvement of the epiphyseal plate was found to be a factor influencing the rate of redisplacement. Of 87 cases with fractured epiphyseal plates a redisplacement rate of only $25.3 \%$ was detected as opposed to $42 \%$ in 288 patients with fractures located away from the physis yielding a significant $P$ value of 0.005 . The total of 375 fractures here is explained by redisplacement due to a new trauma or a defect cast. Another influencing factor is the direction of initial displacement as $42.8 \%$ of dorsally displaced fractures revealed redisplacement while only $20.6 \%$ of fractures with volar angulation did.

Furthermore, $49.1 \%$ of patients in our study presented with an intact ulna and $33.2 \%$ of these displaced secondarily and only $44.2 \%$ needed further treatment. However, $42.5 \%$ of complete fractures showed secondary displacement so that we cannot confirm other studies that consider an intact ulna a risk factor for redisplacement.

\section{Discussion}

The aim of our study was to investigate on a large-scale if closed reduction and immobilization of displaced distal radial fractures provide a sufficient treatment for children and to establish parameters that reveal an increased risk for redisplacement and indicate for Kirschner wire fixation.
Of 393 fractures that we investigated, 263 cases were indicated to be reduced and immobilized because they did not apply for mere immobilization due to their displacement beyond the limits of remodeling. To avoid any bias, we registered any change in angulation or translation after reduction in our study. Since only $14.4 \%$ of these fractures needed further treatment because of redisplacement and the outcome was good, we conclude that closed reduction and immobilization is an appropriate method to treat these fractures in children. In comparison to hospitals that generally treat fractures that display angulation or translation beyond the known limits of remodeling with Kirschner wire fixation to avoid the risk of loss of reduction, in our study $85.6 \%$ of patients were spared general anesthesia as well as hospitalization for hardware removal and the risk of wound infection.

Due to the absence of consent about the remodeling capacity [18-22] very strict limits of remodeling were chosen as described in the methods section. Limits of remodeling were considered $\leq 30^{\circ}$ angulation and $\leq$ shaft width translation for children under 12 years of age and $\leq 15^{\circ}$ angulation and $\leq 1 / 2$ shaft width translation for children aged 12 years or older, respectively. Nevertheless, most fractures were found to be within these strict limits after closed reduction and immobilization which underlines the usefulness of closed reduction and immobilization as the standard treatment for distal radial fractures in children.

However, to optimize treatment of the cases that needed secondary therapy, we analyzed operation reports and intraoperative $\mathrm{X}$-rays amongst other factors increasing the chance of redisplacement. Interestingly, in nearly half of these cases, reports and X-rays revealed that fractures were not optimally reduced since residual angulation of more than $5^{\circ}$ or/and translation of more than corticalis width was accepted during the intervention or more than one attempt of reduction was needed. As it is well-known that operative reports not always contain every detail during a procedure it is even possible that these problems evolve in a greater percentage of cases leading to loss of reduction in need of further treatment. In our study, these aboveexplained difficulties were noted in 17 cases of which five needed secondary Kirschner wire fixation and 12 required
Table 3 Rate of redisplacement of the different fracture types

\begin{tabular}{lcc}
\hline Type of displaced fracture & Number of fractures & Redisplacement rate $(\%)$ \\
\hline Distal radial fracture without special features & 286 & 37.8 \\
Open fracture Gustilo grade I/II & 8 & 26.7 \\
Aitken fracture I & 78 & 26.3 \\
Aitken fracture 0 & 4 & - \\
Green stick fracture & 14 & 24.9 \\
Plurifragmentary fracture & 3 & - \\
Total & 393 & \\
\hline
\end{tabular}


secondary reduction. Furthermore, in 48 more cases, problems during reduction were logged, and $43.8 \%$ of these redisplaced, however, measurements were within the limits of remodeling and patients did not need secondary therapy. Although only $26.2 \%$ of fractures with explicitly reported problems during reduction were reduced again or fixated with Kirschner wires, $58.6 \%$ of fractures with difficulties during reduction revealed loss of reduction. Therefore, we consider the mentioned reported difficulties a risk factor for redisplacement and recommend primary Kirschner wire fixation for these cases to reduce the fraction of $14.4 \%$ of fractures that needed secondary treatment.

Furthermore, our results regarding residual displacement as a risk factor for redisplacement are in agreement with earlier studies $[6,10,13,23-26]$. Strikingly, repeated attempts of reduction have not been described in this context so far. On the other hand, another finding underlines our statement, since only $6.9 \%$ of fractures without any reported problems or without residual displacement needed further treatment and this number does not take into account when repeated attempts of reduction were not noted in the reports possibly due to lack of detailed description of a routine procedure.

Additionally, fractures with involvement of the epiphyseal plate were more resistant to redisplacement pointing to a higher stability of distal fractures. However, we cannot confirm results from some studies that hypothesize that an intact ulna increases the rate of secondary displacement $[11,15,24,27,28]$ as opposed to others that found no influence of an intact ulna on the rate of redisplacement $[15,28]$ since our study found a tendency of increased redisplacement risk that is associated with a fractured ulna. Notably, when using the term "intact ulna" we did not include ulnar buckle fractures or ulnar styloid fractures in this term as opposed to other groups [24].

Only 25 patients in our study were treated with primary Kirschner wire fixation. Indication for this operation was instability during reduction or need of open reduction. The outcome was highly satisfying since only one patient needed further treatment and complication rate was also low since we detected only one case of wound infection and one patient with skin maceration due to Kirschner wires. However, as mentioned above, further hospitalization and anesthesia was necessary for hardware removal so that we confirm our recommendation to keep this method back for the explained selected cases. Furthermore, a recent study has shown that there is no long-term benefit of Kirschner wire fixation regarding the outcome of these fractures in children [29].

Limitations of this study are the short follow-up time of mostly 4-6 weeks so that we can not draw any conclusions on the long-term outcome of the fractures. However, the remodeling potential and long term outcomes after radial fractures in children have been investigated in previous studies [18-22] whereas our goal was to investigate the redisplacement rate after closed reduction without osteosynthesis of fractures in children and its risk factors to avoid this complication and to evaluate this method of treatment. Nevertheless, a follow-up study addressing the group of patients with residual deformities has been planned. Another point to consider is, of course, the retrospective design of the study. However, performing a prospective study with children regarding treatment in an emergency setting will raise ethical concerns and recruitment might be severely prolonged due to the anxiety of the parents. Furthermore, limitating, this has been a single-center study, collecting data from various centers might be a task for the future. Additionally, 7 different attendings and 14 different residents have performed the analyzed reductions, but the large number of cases should compensate for this inhomogeneity. Also, we investigated different fracture types, however, this was considered in the analysis. A further interesting follow-up trial might be an analysis in our institution regarding the impact of the recommended change in the management of the mentioned distal radial fractures on the rate of secondary displacements.

This study presents a large series of distal displaced radial fractures in children. In conclusion, $85.4 \%$ of cases with primary closed reduction and immobilization did not need any further intervention while $10.7 \%$ of reduced fractures needed secondary reduction and $3.8 \%$ of reduced cases were treated with secondary Kirschner wire fixation. Due to our analysis of operative reports, we consider repeated reductions and residual displacement of angulation of $5^{\circ}$ or more and translation of corticalis thickness or more a major risk factor for redisplacement and suggest primary Kirschner wire fixation in these cases.

Conflict of interest Danielle Wendling-Keim, Hans-Georg Dietz and Barbara Wieser declare that they have no conflict of interest.

Compliance with ethical requirements The study was performed according to the ethical requirements; this article does not contain any experiments with human or animal subjects performed by any of the authors.

\section{References}

1. Cheng JC, Shen WY. Limb fracture pattern in different pediatric age groups: a study of 3,350 children. J Orthop Trauma. 1993;7(1):15-22.

2. Galano GJ, Vitale MA, Kessler MW, Hyman JE, Vitale MG. The most frequent traumatic orthopaedic injuries from a national pediatric inpatient population. J Pediatr Orthop. 2005;25(1):39-44.

3. Rodriguez-Merchan EC. Pediatric fractures of the forearm. Clin Orthop Relat Res. 2005;432:65-72.

4. Pretell MJ, Beck N, Brewer J, Baldwin K, Sankar W, Flynn J. Distal metaphyseal radius fractures in children following closed 
reduction and casting: can loss of reduction be predicted? Int Orthop. 2012;36(7):1435-40.

5. Hove LM, Brudvik C. Displaced paediatric fractures of the distal radius. Arch Orthop Trauma Surg. 2008;128(1):55-60.

6. Devalia KL, Asaad SS, Kakkar R. Risk of redisplacement after first successful reduction in paediatric distal radius fractures: sensitivity assessment of casting indices. J Pediatr Orthop B. 2011;20(6):376-81.

7. Miller BS, Taylor B, Widmann RF, Bae DS, Snyder BD, Waters PM. Cast immobilization versus percutaneous pin fixation of displaced distal radius fractures in children: a prospective, randomized study. J Pediatr Orthop. 2005;25(4):490-4.

8. McLauchlan GJ, Cowan B, Annan IH, Robb JE. Management of completely displaced metaphyseal fractures of the distal radius in children. A prospective, randomised controlled trial. J Bone Jt Surg Br. 2002;84(3):413-7.

9. Alemdaroglu KB, Iltar S, Cimen O, Uysal M, Alagoz E, Atlihan D. Risk factors in redisplacement of distal radial fractures in children. J Bone Jt Surg Am. 2008;90(6):1224-30.

10. Proctor MT, Moore DJ, Paterson JM. Redisplacement after manipulation of distal radial fractures in children. J Bone Jt Surg Br. 1993;75(3):453-4.

11. Gibbons CL, Woods DA, Pailthorpe C, Carr AJ, Worlock P. The management of isolated distal radius fractures in children. J Pediatr Orthop. 1994;14(2):207-10.

12. Van Leemput W, De Ridder K. Distal metaphyseal radius fractures in children: reduction with or without pinning. Acta Orthop Belg. 2009;75(3):306-9.

13. Haddad FS, Williams RL. Forearm fractures in children: avoiding redisplacement. Injury. 1995;26(10):691-2.

14. Mani GV, Hui PW, Cheng JC. Translation of the radius as a predictor of outcome in distal radial fractures of children. J Bone Jt Surg Br. 1993;75(5):808-11.

15. Zamzam MM, Khoshhal KI. Displaced fracture of the distal radius in children: factors responsible for redisplacement after closed reduction. J Bone Jt Surg Br. 2005;87(6):841-3.

16. Flynn JM, Sarwark JF, Waters PM, Bae DS, Lemke LP. The surgical management of pediatric fractures of the upper extremity. Instr Course Lect. 2003;52:635-45.

17. Choi KY, Chan WS, Lam TP, Cheng JC. Percutaneous Kirschner-wire pinning for severely displaced distal radial fractures in children. A report of 157 cases. J Bone Jt Surg Br. 1995;77(5):797-801.

18. Zimmermann R, Gschwentner M, Kralinger F, Arora R, Gabl M, Pechlaner S. Long-term results following pediatric distal forearm fractures. Arch Orthop Trauma Surg. 2004;124(3):179-86.

19. Zimmermann R, Gschwentner M, Pechlaner S, Gabl M. Remodeling capacity and functional outcome of palmarly versus dorsally displaced pediatric radius fractures in the distal one-third. Arch Orthop Trauma Surg. 2004;124(1):42-8.

20. Ploegmakers JJ, Verheyen CC. Acceptance of angulation in the non-operative treatment of paediatric forearm fractures. J Pediatr Orthop B. 2006;15(6):428-32.

21. Linhart WE, von Laer L. General considerations in the management of paediatric injuries. Orthopade. 2005;34(11):1169-84.

22. Schmittenbecher PP, Dietz HG, Uhl S. Spätergebnisse nach Unterarmfrakturen im Kindesalter. Unfallchirurg. 1991;94(4):186-90.

23. Jordan RW, Westacott DJ. Displaced paediatric distal radius fractures-when should we use percutaneous wires? Injury. 2012;43(6):908-11.

24. Sankar WN, Beck NA, Brewer JM, Baldwin KD, Pretell JA. Isolated distal radial metaphyseal fractures with an intact ulna: risk factors for loss of reduction. J Child Orthop. 2011;5(6):459-64.

25. Fenton P, Nightingale P, Hodson J, Luscombe J. Factors in redisplacement of paediatric distal radius fractures. J Pediatr Orthop B. 2012;21(2):127-30.

26. Hang JR, Hutchinson AF, Hau RC. Risk factors associated with loss of position after closed reduction of distal radial fractures in children. J Pediatr Orthop. 2011;31(5):501-6.

27. Roy DR. Completely displaced distal radius fractures with intact ulnas in children. Orthopedics. 1989;12(8):1089-92.

28. Monga P, Raghupathy A, Courtman NH. Factors affecting remanipulation in paediatric forearm fractures. J Pediatr Orthop B. 2010;19(2):181-7.

29. Ozcan M, Memisoglu S, Copuroglu C, Saridogan K. Percutaneous Kirschner Wire fixation in distal radius metaphyseal fractures in children: does it change the overall outcome? Hippokratia. 2010;14(4):265-70. 